\title{
A Deterministic Computational Procedure for Space Environment Electron Transport
}

\author{
John E. Nealy ${ }^{\mathrm{a}}$, C. K. Chang ${ }^{\mathrm{b}}$, Ryan B. Norman ${ }^{\mathrm{c}, *}$, Steve R. Blattnig ${ }^{\mathrm{c}}$, Francis F. Badavi ${ }^{\mathrm{b}}$, Anne M. Adamczyk ${ }^{\mathrm{d}}$ \\ ${ }^{a}$ Old Dominion Research Foundation, Norfolk, Virginia 23508, USA \\ ${ }^{b}$ Christopher Newport University, Newport News, Virginia 23606, USA \\ ${ }^{c}$ NASA Langley Research Center, Hampton, Virginia 23681, USA \\ ${ }^{d}$ University of Tennessee, Knoxville, Tennessee 37996, USA
}

\begin{abstract}
A deterministic computational procedure for describing the transport of electrons in condensed media is formulated to simulate the effects and exposures from spectral distributions typical of electrons trapped in planetary magnetic fields. The primary purpose for developing the procedure is to provide a means of rapidly performing numerous repetitive transport calculations essential for electron radiation exposure assessments for complex space structures. The present code utilizes well-established theoretical representations to describe the relevant interactions and transport processes. A combined mean free path and average trajectory approach is used in the transport formalism. For typical space environment spectra, several favorable comparisons with Monte Carlo calculations are made which have indicated that accuracy is not compromised at the expense of the computational speed.
\end{abstract}

Key words: Space radiation, Planetary environments, Electrons, Absorbed dose, LEO

PACS: 07.87.+v, 28.41.Qb, 94.20.wq

\section{Introduction}

The concept of developing a rapid analysis electron transport code at NASA Langley Research Center (LaRC) arose from a desire to have a companion code for the LaRC deterministic transport code HZETRN 11. The algorithm developed here for electron transport is based on the Continuous Slowing Down Approximation (CSDA) combined with an elastic multiple scattering formulation to define an electron mean free path and a transmission function at a given target location. The cross sections relevant to CSDA and multiple scattering are described in the following section. The pertinent cross sections are calculated with well-established theoretical formulas, valid over the energy range representative of trapped electrons in planetary magnetic fields. Consequently, a calculation of positron production and annihilation is incorporated. The cross section equations are cast in terms of specific elemental atomic species, from which cross sections applicable to any user defined molecular system (mono or polyatomic) are automatically constructed.

Details of the transport formulation are described in a subsequent section, and are based on motion related to some initial direction (axis of propagation). Behavior of slowing and stopping of electrons and their associated

*Corresponding author. Address: NASA Langley Research Center, MS 188E, Hampton, VA 23681. Tel.: +1 (757) 864 2185; fax: +1 (757) 8648911

Email addresses: Ryan.B.Norman@nasa.gov (Ryan B. Norman ) bremsstrahlung is evaluated in terms of quantities contained in the direction of the hemisphere centered on the initial direction. The scattering and mean free path calculations permit definition of mean trajectories relative to the initial motion direction, so that axisymmetric spatial and energy distributions may be inferred.

Comparisons with the corresponding Monte Carlo (MC) calculations are shown. The MC cases utilized several hours of machine time, whereas the LaRC calculations were practically instantaneous. Despite some difference in final magnitudes, general functional behavior is consistent in the results. Consequently, the deterministic code can be reliably used in trade studies where rapid analyses are necessary.

The present code has the desirable feature that the required inputs are user defined through files specifying material composition along with the incident environment differential energy spectra for electrons and photons. Also included with the code is a permanent atomic database file which details the pertinent atomic properties. Most results in this paper are presented for three selected materials: aluminum $(\mathrm{Al})$, tantalum $(\mathrm{Ta})$, and water $\left(\mathrm{H}_{2} \mathrm{O}\right)$. This selection of target materials was chosen to provide examples of light and heavy materials in addition to examples of elemental and compound materials. The materials were also selected for their uses in space applications. Aluminum is a common spacecraft structural material, water closely approximates human tissue, and tantalum is used in shielding sensitive electronics from electron and photon 
exposure in the planetary trapped radiation belts.

\section{Cross Sections}

The theoretical description of propagation of energetic electrons and photons in condensed media requires some representation of interaction cross sections with constituent atoms of the medium. Application of principles of quantum and semi-classical physics have gone far toward providing formulations for electron and photon interaction cross sections. Although many of these formulations are mathematically complicated, they are often amenable to approximation and parameterizations that greatly simplify their practical application. The following subsections describe the formulations used here for interactions of electrons and photons.

\subsection{Electrons}

Free energetic electrons passing through a material are slowed and may eventually be stopped as they interact with the fields of the nuclei and bound electrons of the material. Interactions also result in a change of direction (scattering). In the present analysis, the electron deceleration process is expressed in terms of energy loss by collisions and the accompanying photon production (bremsstrahlung). The dominant scattering process is assumed to be that of small angle elastic (Rutherford) scattering and is the only scattering process taken into account in the present formulation.

\subsubsection{Collisional Slowing and Stopping}

Electron deceleration by collision processes, in which energy from the projectile electron is imparted to the electrons of the medium, is usually cast in terms of the stopping power, $S$, which is defined as the energy loss per unit scaled distance, $-d E / d x$. Note that the distance $x$ is measured in units of $\mathrm{g} / \mathrm{cm}^{2}$. The resultant expression for the collisional stopping power has been often referred to as the modified Bethe-Bloch formula and is given here in the notation of Anderson [2],

$$
S_{\mathrm{col}} \equiv-\frac{d E}{d x}=2 \pi r_{e}^{2} m_{e} c^{2} \rho^{\prime}\left(N_{0} Z / A\right) G_{T} C^{ \pm},
$$

where

$$
G_{T}=\frac{\left(T+m_{e} c^{2}\right)^{2}}{T\left(T+2 m_{e} c^{2}\right)}
$$

and

$$
C^{ \pm}=\left\{\ln \left[\frac{2\left(T+2 m_{e} c^{2}\right)}{m_{e} c^{2}\left(I / m_{e} c^{2}\right)^{2}}\right]+F^{ \pm}-\delta-\lambda\right\}
$$

The superscript $( \pm)$ indicates applicability to both electrons (-) and positrons $(+)$. Here, $r_{e}$ is the classical electron radius, $m_{e}$ is the rest mass of the electron, $c$ is the speed of light in vacuum, $\rho^{\prime}$ is the scaled mass density with distance measured in $\mathrm{g} / \mathrm{cm}^{2}, N_{0}$ is Avogadro's constant, $Z$ is the atomic number of the material, $A$ is the atomic mass of the material, $T$ is the kinetic energy of the electron or positron, $I$ is the mean atomic ionization potential, $\delta$ is the density correction term, and $\lambda$ is the shell correction term. The $F^{ \pm}$quantity in Eq. (2) is a function only of projectile energy and is slightly different (a few percent) for electrons and positrons. The present formulation implements only the $F^{ \pm}$function that applies to electrons. It may be written as the sum of three terms [2],

$$
F^{ \pm} \approx F^{-}=f_{1}+f_{2}-f_{3},
$$

where

$$
\begin{aligned}
& f_{1}=2+2 \ln \left[\frac{\Delta(T}{2 m_{e} c^{2}}\right] \\
& f_{2}=\frac{T^{2} / 8+m_{e} c^{2}\left(2 T+m_{e} c^{2}\right) \ln \left(\frac{1}{2}\right)}{\left(T+m_{e} c^{2}\right)^{2}},
\end{aligned}
$$

and

$$
f_{3}=1+\frac{T\left(T+2 m_{e} c^{2}\right)}{\left(T+m_{e} c^{2}\right)^{2}} .
$$

The quantity $\delta$ in Eq. (2) represents a modification to the Bethe-Bloch stopping power usually referred to as the "density correction." For this work, a parameterized form of $\delta$ based on extensive experimental data [3] is used.

The remaining correction term, $\lambda$, in Eq. (2), is called the "shell correction" and is only important at low energies when the projectile electron speed, $v$, approaches the speed of a bound electron, $v_{I}$. Its value, as used in the present calculations, is given by 4

$$
\lambda \approx\left(\frac{v_{I}}{v}\right)^{2} \approx \frac{I\left(I+2 m_{e} c^{2}\right)\left(T+m_{e} c^{2}\right)^{2}}{T\left(T+2 m_{e} c^{2}\right)\left(I+m_{e} c^{2}\right)^{2}} .
$$

It is seen in Eq. (1) that $S_{\text {col }}$ is unbounded as $T \rightarrow 0$ due to the $G_{T}$ factor. To correct the unbounded behavior as $T \rightarrow 0$, the non-relativistic stopping power derivation provided by Bohr and discussed in the paper by Sigmund [4] can be used. Bohr's derivation results in a stopping power that approaches zero at very low energies and exhibits a maximum. The Bohr formula may be written in CGS (centimeters-grams-seconds) units as

$$
S_{\mathrm{Bohr}}=\frac{4 \pi n e^{4}}{m_{e} v^{2}} \ln \left(\frac{C_{\gamma} m_{e} v^{3}}{e^{2} \omega_{I}}\right) \equiv \frac{a}{T} \ln \left(b T^{3 / 2}\right),
$$

with $n=\rho N_{0} / A$ is the number density, $\rho$ is the mass density, $e$ is the electric charge, $C_{\gamma}=2 \mathrm{e}^{-\gamma}, \gamma$ is Euler's constant, $\omega_{I}=I / \hbar$, and $\hbar$ is the reduced Planck's constant. The kinetic energy at which the Bohr formula for stopping power exhibits a maximum is found from $\frac{d S_{\mathrm{Bohr}}}{d T}=0$,

$$
\ln \left(b T_{B}^{3 / 2}\right)=\frac{3}{2}
$$


From Eq. [5],

$$
b=2^{3 / 2} C_{\gamma} /\left(e^{2} \omega_{I} \sqrt{m_{e}}\right)
$$

and

$$
T_{B}=\left(\frac{\exp ^{3 / 2} e^{2} \sqrt{m_{e}} I}{2^{3 / 2} C_{\gamma} \hbar}\right)^{2 / 3},
$$

where $T_{B}$ is the projectile kinetic energy at peak stopping power and exp is the base of natural logarithms. If Eq. (1) is compared with Eq. (5), the natural logarithm term in Eq. (5) can be identified with the $C^{ \pm}$in Eq. (1). This gives

$$
C_{\max }^{ \pm}=\ln \left(b T_{B}^{3 / 2}\right),
$$

and

$$
S_{\max } \equiv 2 \pi r_{e}^{2} m_{e} c^{2} N_{0} G_{T}(Z / A) C_{\max }^{ \pm} .
$$

While Eq. (1) applies to $T \geq T_{B}$, an extension of Bohr's stopping power formula to low energies, for $T<T_{B}$, is provided by Sigmund [4. For the case of $T<T_{B}$, a simple functional fit for the collisional stopping power in units of $\mathrm{MeV} \mathrm{cm} / \mathrm{g}$ has been derived for the present work,

$$
S_{\text {col }}=S_{\max } \exp \left\{-0.179\left[\ln \left(T_{B} / T\right)\right]^{2.05}\right\} .
$$

Three simple materials important to space exploration applications have been selected to demonstrate results of the present model calculations: aluminum, tantalum, and water. These materials were chosen to represent a broad range of atomic properties and material densities. The collision stopping powers, as calculated for these substances over the energy range of the code formulation, were compared with the stopping powers obtained from the database tabulations of the National Institute of Standards and Technology (NIST) [5]. The comparisons indicated that the present formulation is quite acceptable over the energy range of interest for space applications (0.01 $1000 \mathrm{MeV}$ ), with the largest discrepancies occurring at the lowest energies for heavy elements [6].

\subsubsection{Radiative Energy Loss (Bremsstrahlung)}

Accelerating (and decelerating) charged particles lose energy by photon emission. Elaborate quantum-theoretical calculations have gone far toward quantifying these bremsstrahlung cross sections. A detailed description of the theoretical results is given in Koch and Motz [7. The formulation may be greatly simplified by using several parameterizations; the implementation for this work is essentially the same as that for the default cross sections of the EGSnrc Monte Carlo code [8]. Several pertinent parameters that lead to the ultimate cross section formula may be defined as 8 ]

$$
F_{c} \equiv 4\left[\frac{1}{3} \ln (Z)+f_{c}\right],
$$

where

$$
f_{c} \equiv\left\{\begin{array}{ll}
0.0 & E_{\nu}<50 \mathrm{MeV} \\
f_{c c} & E_{\nu} \geq 50 \mathrm{MeV}
\end{array},\right.
$$

with

$$
\begin{aligned}
f_{c c} & \equiv(\alpha Z)^{2} \sum_{\beta=1}^{\infty} \frac{1}{\beta\left(\beta^{2}+\alpha^{2} Z^{2}\right)} \\
& \cong 7.4564 \times 10^{-5} Z^{1.9137} .
\end{aligned}
$$

Here, $E_{\nu}$ is the emitted photon energy and $\alpha$ is the fine structure constant. The term $F_{c}$ serves to provide a high energy Coulomb correction. Another parameter defines the effects of screening in terms of an energy variable [8],

$$
\delta_{s}=\left(\frac{136}{Z^{1 / 3}}\right)\left[\frac{E_{\nu} m_{e} c^{2}}{E_{e}\left(E_{e}-E_{\nu}\right)}\right],
$$

which involves the electron total energy, $E_{e}=T+m_{e} c^{2}$. The screening functions have been specified as 8

$$
\begin{aligned}
& \phi_{1}\left(\delta_{s}\right)=\left\{\begin{array}{ll}
20.867-3.242 \delta_{s}+0.625 \delta_{s}^{2} & \delta_{s} \leq 1 \\
21.12-4.184 \ln \left(\delta_{s}+0.952\right) & \delta_{s}>1
\end{array},\right. \\
& \phi_{2}\left(\delta_{s}\right)=\left\{\begin{array}{ll}
20.209-1.935 \delta_{s}+0.086 \delta_{s}^{2} & \delta_{s} \leq 1 \\
\phi_{1}\left(\delta_{s}\right) & \delta_{s}>1
\end{array} .\right.
\end{aligned}
$$

To account for the triplet production process, a parameter, $\xi$, is introduced which depends upon atomic charge and involves the Coulomb correction above 8 . A parameterized fit for $\xi$ in units of $\mathrm{cm}^{2} / \mathrm{g}$ has been developed for this work as

$$
\xi \approx 1.147 \sin (0.42 \ln Z)+0.12\left\{[\sin (0.76 \ln Z)]^{2}\right\}^{\frac{3}{4}} .
$$

The final expression for the cross section for production of a photon of energy $E_{\nu}$ by interaction of an electron of total energy $E_{e}$ on an atom of charge $Z$ may be written as

$$
\frac{d \sigma_{\nu, e}}{d E_{\nu}}=\frac{\alpha r_{e}^{2}}{E_{\nu}} \frac{N_{0} Z(Z+\xi)}{A} H_{\nu},
$$

where

$$
\begin{aligned}
H_{\nu}= & {\left[1+\left(\frac{E_{e}-E_{\nu}}{E_{e}}\right)^{2}\right]\left(\phi_{1}-F_{c}\right) } \\
& -\frac{2}{3}\left(\frac{E_{e}-E_{\nu}}{E_{e}}\right)\left(\phi_{2}-F_{c}\right) .
\end{aligned}
$$

An obvious energy constraint is that only photons can be produced that have energy less than the initial electron kinetic energy. Consequently, $d \sigma_{\nu, e} / d E_{\nu}$ is calculated at each electron energy $T$, for selected values of $E_{\nu} / T$ : [0.001, $0.05,0.1,0.2,0.3,0.4,0.6,0.8,0.9,0.95,0.98,0.99,1.0]$. This distribution of energy values has been chosen to accurately resolve the cross section variations at both ends 
of the energy spectrum. A source term $\zeta$, for production of photons by an electron flux $\varphi_{e}$, may be written in terms of the bremsstrahlung cross section as

$$
\zeta\left(E_{\nu}, T\right)=\int_{E_{\nu}}^{T} \varphi_{e}\left(T^{\prime}\right) \frac{d \sigma_{\nu, e}}{d E_{\nu}} d T^{\prime} \text { for } E_{\nu} \leq T .
$$

The corresponding energy loss term due to radiative processes may be evaluated as

$$
S_{\mathrm{rad}}=\int_{0}^{E_{\nu}} E_{\nu}^{\prime} \frac{d \sigma_{\nu, e}}{d E_{\nu}^{\prime}} d E_{\nu}^{\prime}
$$

Using Eq. 13), the radiative loss stopping powers for the three materials selected for this study were calculated. In contrast to the collision stopping powers, the bremsstrahlung stopping power is greater for higher charge elements and increases monotonically with energy. The logarithmic stopping power for the present approximate formulation is nearly linear with the logarithm of the energy and begins to have substantial effects for kinetic energies greater than a few MeV. Comparison with NIST [5] data revealed some disparities at low kinetic energies $(T<0.1 \mathrm{MeV})$ 6], but, as will be shown subsequently in Sec. 2.3, bremsstrahlung effects on the general transport process have a significant impact only for kinetic energies greater than several $\mathrm{MeV}$.

\subsubsection{Multiple elastic scattering}

Strict conservation principles require that electron-atom interactions generally result in energy exchange accompanied by re-direction with respect to spatial variables. Approximations that tend to decouple energy loss processes and directional changes greatly simplify electron transport analysis. In the present work, these processes are made practically independent. Energy loss is specified by collision and radiative losses without regard for directional change, while projectile trajectories are described by elastic scattering interactions. Such assumptions call for careful scrutiny with regard to both broad energy spectra and the wide variety of material types. Some precedents have been set in earlier works [9, 10] in which such approaches have been used. In the present formulation, the elastic (Rutherford) scattering cross sections, $\sigma_{\mathrm{R}}$, are implemented as a basis for calculating an electron transport mean free path,

$$
\lambda_{\mathrm{tr}} \equiv \frac{\rho}{n \sigma_{\mathrm{R}}},
$$

where $n=\rho N_{0} / A$ is the number of scattering centers per unit volume in the medium. The present development closely follows that of Kawrakow and Rogers [8, in which the screened Rutherford cross section is given as

$$
\frac{d \sigma_{\mathrm{R}}}{d \theta} \equiv 2 \pi\left[\frac{Z e^{2}\left(T+m_{e} c^{2}\right)}{T\left(T+2 m_{e} c^{2}\right)(1-\cos \theta+1 / \epsilon)}\right]^{2},
$$

where $\theta$ is the scattering angle and $\epsilon$ is a parameterized screening function,

$$
\epsilon \approx 1413\left[\left(1+T / m_{e} c^{2}\right)^{2}-1\right](Z \alpha)^{-2 / 3} .
$$

Integrating Eq. (15) over all scattering angles to obtain the total cross section allows the electron transport mean free path to be written as [6]

$$
\begin{aligned}
\lambda_{\mathrm{tr}} & \equiv \rho / n \sigma_{R} \\
& =\frac{A}{2 \pi N_{0} e^{4} Z^{2}} \frac{\left(T^{2}+2 T m_{e} c^{2}\right)^{2}}{[\ln (2 \epsilon)-1]\left(T+m_{e} c^{2}\right)^{2}} .
\end{aligned}
$$

The above quantity will be dealt with in the subsequent section on the transport algorithm. Note that the behavior of the transport mean free path increases monotonically with kinetic energy and is nearly logarithmically linear with the log of the kinetic energy, having generally higher values for lower atomic weight elements.

Another parameter related to elastic multiple scattering is the mass scattering power [11]. This quantity is analogous to stopping power, but refers to solid angle scattering rather than energy loss. The mass scattering power is defined in Li and Rodgers 11 as

$$
P_{s} \equiv \pi\left[\frac{2 r_{e} m_{e}^{2} c^{4} Z\left(T+m_{e} c^{2}\right)}{T\left(T+2 m_{e} c^{2}\right)}\right]^{2} \frac{N_{0}}{Z} Y\left(\theta_{r}\right),
$$

where

$$
Y\left(\theta_{r}\right) \equiv \ln \left(1+\theta_{r}^{2}\right)-1+\frac{1}{1+\theta_{r}^{2}}
$$

with

$$
\theta_{r}=\frac{\sqrt{T\left(T+2 m_{e} c^{2}\right)}}{\alpha m_{e} c^{2} Z^{1 / 3}} .
$$

The mass scattering power is used in the transport algorithm to relate electron traversal on a specific trajectory to the axial penetration distance. The above formulation predicts a nearly linear monotonically decreasing variation of the logarithm of scattering power with the logarithm of energy.

\subsection{Photons}

Bremsstrahlung photons produced by electron-atom interactions are attenuated and absorbed in the transport medium. In the present work, only the three most important processes involved in photon energy degradation and absorption are considered: photoelectric absorption, incoherent scattering, and electron-positron pair production. These processes are dominant for different energy ranges, but when taken collectively, they closely approximate the total attenuation for the energy range considered here $(0.001-1000 \mathrm{MeV})$. The contribution due to coherent (or Rayleigh) scattering has not been included in this analysis since it does not significantly add to the total cross section over the energy range of interest (0.001 - 1000 $\mathrm{MeV})$. 


\subsubsection{Photoelectric Absorption}

Photoelectric absorption involves interactions of a photon with a bound electron in which the photon energy is absorbed with a corresponding kinetic energy increase for the electron. The detailed evaluation of the photoelectric cross sections requires the use of complicated atomic models in sophisticated solutions of the Schrödinger equation for all energies and elements under consideration. For the present formulation, liberal use is made of parametric fitting formulas devised from the extensive calculations and tabulations provided by NIST [5]. First, the energy values for the absorption discontinuities of the $K$ and $L$ shells have been approximated as

$$
\begin{aligned}
& E_{K} \approx 5.435 \times 10^{-3} Z^{2.2038} \mathrm{keV} \quad(1 \leq Z \leq 92), \\
& E_{L} \approx 1.5754 \times 10^{-4} Z^{2.5984} \mathrm{keV} \quad(30 \leq Z \leq 92) .
\end{aligned}
$$

Note that energies in this section are in $\mathrm{keV}$, and $L$-edge energies are only considered for $Z \geq 30$. In addition, only the discontinuities due to the $K$ and $L$ shells have been considered. The discontinuities (edges) due to the electronic shells occur at values of the photon energy corresponding to the binding energies of electrons involved in the interaction. When the incoming photon has an energy greater than the binding energy of a given atomic shell or subshell, a discontinuity may occur.

At the edge discontinuities, the NIST data has also been used to develop formulas for the upper $(u)$ and lower $(l)$ values of the photoelectric cross section:

$$
\begin{array}{rlrl}
\sigma_{K u} & =7165.5 E_{K}^{-1.5474} & & \mathrm{~cm}^{2} / \mathrm{g}, \\
\sigma_{K l} & =593.59 E_{K}^{-1.327} & \mathrm{~cm}^{2} / \mathrm{g} \\
\sigma_{L u} & =9620.6 E_{L}^{-1.5113} & \mathrm{~cm}^{2} / \mathrm{g}, \\
\sigma_{L l} & =1633.5 E_{L}^{-1.2124} & \mathrm{~cm}^{2} / \mathrm{g} .
\end{array}
$$

Examination of typical photoelectric cross sections reveals that the logarithmic slope,

$$
m \equiv \frac{d\left(\ln \sigma_{\mathrm{PE}}\right)}{d\left(\ln E_{\nu}\right)}
$$

is very nearly constant for a given atomic system between absorption edges. Using NIST 5] cross section data, parametric fits were used to approximate the logarithmic slopes.

$$
\begin{aligned}
& \text { For } E_{\nu}>E_{K}: \\
& m_{K}=\left\{\begin{array}{ll}
6.9068 \times 10^{-3} Z-3.014 & Z \leq 10 \\
-3.3147 Z^{-0.08156} & Z \geq 11
\end{array} .\right.
\end{aligned}
$$

For $.1 \mathrm{keV} \leq E_{\nu} \leq E_{K}$ :

$$
m_{L}=\frac{\ln \left(\frac{\sigma_{K l}}{\sigma_{L u}}\right)}{\ln \left(\frac{E_{K}}{E_{L}}\right)} .
$$

For all $Z$ and $E_{K}<E_{\nu}<10000 \mathrm{keV}$ :

$$
\sigma_{\mathrm{PE}}=\sigma_{K u}\left(\frac{E_{\nu}}{E_{K}}\right)^{m_{K}} .
$$

For $Z \geq 29$ and $E_{L}<E_{\nu}<E_{K}$ :

$$
\sigma_{\mathrm{PE}}=\sigma_{L u}\left(\frac{E_{\nu}}{E_{L}}\right)^{m_{L}}
$$

An extension to energies less than the lowest edge energy considered was extrapolated with a power law function approaching an asymptote based on an extrapolated maximum absorption cross section. For $Z \leq 29$ and for energies below the $K$-edge, an asymptotic cross section is calculated using the average ionization potential,

$$
\sigma_{I}=\sigma_{K l}\left(\frac{I}{E_{K}}\right)^{m_{K}}
$$

The extrapolation formula is then

$$
\sigma_{\mathrm{PE}}=\frac{\sigma_{I}}{1+A_{P} E_{\nu}^{p}}
$$

where the exponent $p$ and coefficient $A_{P}$ are given by

$$
p=\frac{m_{K}}{\left(\sigma_{K l} / \sigma_{I}\right)-1} ; \quad A_{P}=\frac{\left(\sigma_{I} / \sigma_{K l}\right)-1}{E_{K}^{p}} .
$$

These constants are found by matching the cross section and the slope at the $K$-edge. For $Z \geq 29$, the low energy extrapolation below the $L$-edge is found by using an $L$ subscript instead of $K$ in Eqs. $222-(26)$.

For the three selected materials, the photoelectric cross sections, as calculated with the present parameterization were compared with their NIST 5 counterparts and agreements were considered to be fair to good [6].

\subsubsection{Inelastic Photon Interactions}

As the photoelectric absorption coefficient decreases rapidly with increasing energy, the next process to dominate is when the photon imparts a portion of its energy to an electron resulting in scattering of a lower energy photon. The process is referred to as inelastic (incoherent) scattering. The cross section formula for release of an electron of kinetic energy $T_{e}$ by a photon of initial energy $E_{\nu}$ has been taken from Anderson [2] as the free electron Klein-Nishina process. The energy constraints are $0 \leq T_{e} \leq T_{\max }$ where

$$
T_{\max }=\frac{2 E_{\nu}^{2}}{m_{e} c^{2}+2 E_{\nu}} .
$$

Here, $T_{e}$ is the kinetic energy of the emitted electron and $E_{\nu}$ is the incident photon energy. The cross section formulation is

$$
\frac{d \sigma_{\mathrm{KN}}}{d T_{e}}=\frac{N_{0} Z}{A} \frac{\pi r_{e}^{2}}{q E_{\nu}}\left[2+\left(\frac{R^{\prime}}{q}\right)^{2}+\frac{R^{\prime} T_{e}}{E_{\nu}}-\frac{2}{q R^{\prime}}\right]
$$


with

$$
R^{\prime} \equiv \frac{T_{e}}{E_{\nu}-T_{e}} \text { and } q \equiv \frac{E_{\nu}}{m_{e} c^{2}} .
$$

In order to render the cross section applicable to bound atomic electrons, elaborate quantum theory calculations are required. Such considerations are only appreciable at lower photon energies. For this work, a parametric multiplying function has been developed to approximate this effect,

$$
\ln \Phi\left(Z, E_{\nu}\right)=-\left[\frac{B_{1} \ln (Z)+B_{2}}{E_{\nu}}\right]^{\left[B_{3}-B_{4} \ln (Z)\right]},
$$

where $B_{1}=0.471, B_{2}=1.5184, B_{3}=1.003$, and $B_{4}=0.13317$ when $E_{\nu}$ is given in units of $\mathrm{MeV}$. The final inelastic scattering cross section then becomes:

$$
\frac{d \sigma_{\mathrm{in}}}{d T_{e}}=\Phi\left(Z, E_{\nu}\right) \frac{d \sigma_{\mathrm{KN}}}{d T_{e}} .
$$

For each $E_{\nu}$, the total inelastic cross section is found by integration over the appropriate emitted electron energy;

$$
\sigma_{\text {in }}=\int_{0}^{T_{\max }} \frac{d \sigma_{\text {in }}}{d T^{\prime}} d T^{\prime} .
$$

As a function of incident photon energy, the total inelastic cross sections, calculated with the present model, were compared with the corresponding NIST [5] values. The model approximation was in better agreement for lighter materials. An exception occurred at higher energies where the model formulas are slightly inaccurate 6]. However, in this energy range, the inelastic cross section does not contribute appreciably to the total photon attenuation, as will be subsequently shown in Sec. 2.3 .

\subsubsection{Pair Production}

Electron-positron pair production may occur when a photon of sufficient energy interacts with a strong localized Coulomb field. The photon initial energy is transformed into the combined rest mass of the newly created particles $\left(2 m_{e} c^{2}\right)$, with any remaining energy appearing as kinetic energy of the new particles. A comprehensive description of the process may be found in Motz et al. [12, where a variety of cross section quantities are derived for several aspects of the phenomenon. The formulation from $\mathrm{Li}$ and Rogers [1], chosen for use in the present work, applies to the cross section for production of a positron of total energy $E_{+}$by a photon of initial energy $E_{\nu}$. Only positron production associated with pair production is considered in this work. The positron production differential cross section may be written in simple terms as

$$
\frac{d \sigma_{\mathrm{pp}}}{d E_{+}}=\frac{N_{0}}{A} \frac{\alpha r_{e}^{2} Z(Z+\xi)}{E_{\nu}^{3}}\left(Q_{1}+Q_{2}\right),
$$

where

$$
Q_{1}=\left[E_{+}^{2}+\left(E_{\nu}-E_{+}\right)^{2}\right]\left[\phi_{1}-F_{c}\right]
$$

and

$$
Q_{2}=\frac{2}{3} E_{+}\left(E_{\nu}-E_{+}\right)\left[\phi_{2}-F_{c}\right]
$$

The terms $\xi, \phi_{1}\left(\delta^{\prime}\right), \phi_{2}\left(\delta^{\prime}\right)$, and $F_{c}$ are the same as for the bremsstrahlung cross section, Eqs. (7)-110, with the exception that the value of $\delta^{\prime}$ is determined as

$$
\delta^{\prime}=\frac{136 m_{e} c^{2} E_{\nu}}{Z^{1 / 3} E_{+}\left(E_{\nu}-E_{+}\right)} .
$$

Energy conservation constrains the positron energy as

$$
m_{e} c^{2} \leq E_{+} \leq T_{\max }+m_{e} c^{2}
$$

with

$$
T_{\max }=E_{\nu}-2 m_{e} c^{2}
$$

The above formulas for pair production become somewhat inaccurate as the pair production threshold is approached. Consequently, a power law function is fit at the first energy grid value greater than $4 \mathrm{MeV}\left(\equiv E_{4}\right.$, see below). Now, the low energy pair production cross section is given as

$$
\sigma_{\mathrm{pp}}=A_{\mathrm{pp}}\left(E_{\nu}-1.03\right)^{y} \text { for } 1.03 \mathrm{MeV}<E_{\nu}<E_{4}
$$

with

$$
y=\left.\frac{d \sigma_{\mathrm{pp}}}{d E_{+}}\right|_{E_{+}=E_{4}} \frac{E_{4}-1.03}{\sigma_{\mathrm{pp}}\left(E_{4}\right)},
$$

and

$$
A_{\mathrm{pp}}=\frac{\sigma_{\mathrm{pp}}\left(E_{4}\right)}{\left(E_{4}-1.03\right)^{y}}
$$

A source term for positron production may be derived in a manner similar to that described for production of bremsstrahlung photons. See Eq. 12). For a photon differential flux spectrum $\varphi_{\nu}\left(E_{\nu}, x\right)$, positron production may occur between energies $E_{\mathrm{pp} \text {,min }}=2 m_{e} c^{2}$ and $E_{\mathrm{pp}, \max }=E_{\nu, \max }-2 m_{e} c^{2}$, where $E_{\nu, \max }$ is the highest photon energy value considered. The expression for the source term becomes

$$
\zeta\left(E_{+}, x\right)=\int_{E_{\mathrm{pp}, \min }}^{E_{\mathrm{pp}, \max }} \varphi_{\nu}\left(E_{\nu}^{\prime}, x\right) \frac{d \sigma_{\mathrm{pp}}}{d E_{+}} d E_{\nu}^{\prime} .
$$

It should be noted that in parallel with the positron production process, a similar cross section expression applies 
to the creation of the partner electron, along with the corresponding source term for secondary electrons. Just as for the inelastic process, the pair production cross section may be found by integrating Eq. (31) over the allowed positron energy ranges, namely from $m_{e} c^{2}$ to $E_{\nu}-m_{e} c^{2}$.

Results of pair production cross sections, as calculated from the present model were compared with the appropriate NIST 5 values. Agreement was very good except for energies near the threshold region where the power law extrapolation formula was invoked [6].

\subsection{Cross Section Implementation}

The individual cross sections described previously are used in combination to provide three critical parameters essential for description of the electron-photon transport process. The ultimate slowing and stopping of electrons is governed by the total stopping power obtained from the sum of the collision and radiative stopping powers. The collision process dominates at low energies (less than approximately $1 \mathrm{MeV}$ ). Here, the radiative process assumes the dominant role at high energies (greater than approximately $1 \mathrm{MeV}$ ). A transitional minimum occurs in the neighborhood of $1 \mathrm{MeV}$ where the lower energy inaccuracies in the radiative stopping power of the present model have insignificant impact on the total stopping power.

The general attenuation and extinction of photons in a medium is found to be closely related to the sum of the photoelectric, inelastic, and pair production cross sections. In the present model, the coherent (Rayleigh) scattering processes were not considered because of their relative unimportance in space radiation effects. The inaccuracies in the inelastic cross sections at energies larger than approximately $100 \mathrm{MeV}$ have insignificant impact on the total attenuation of photons due to the pair production process dominating at higher energies [6].

The final parameter of importance to the electronphoton transport process is that of the photon energy deposition coefficient which is used to calculate effective photon dose. See Eq. 63) in Sec. 4.1. This relates to those fractions of the photon attenuation processes $\left(f_{\mathrm{PE}}, f_{\mathrm{in}}\right.$, $\left.f_{\mathrm{pp}}\right)$ that produce secondary electrons. For photoelectric absorption, it is assumed that the secondary electron is emitted with the same energy as the incident photon (i.e. the binding energy of the released electron is neglected) and the value of $f_{\mathrm{PE}}$ is unity. In the case of inelastic scattering [13],

$$
f_{\text {in }}=1-\frac{E_{\nu, \text { in }}}{E_{\nu}}
$$

where $E_{\nu \text {,in }}$ is the energy of the photon produced in the inelastic process initiated by a photon of energy $E_{\nu}$. For the pair production process, $f_{\mathrm{pp}}=1-2 m_{e} c^{2} / E_{\nu}$.

An additional reduction in the photon energy deposition coefficient is required to discount secondary electrons that subsequently produce additional bremsstrahlung. In the present model, this factor is represented by $\left(1-S_{\text {rad }} / S_{\text {Tot }}\right)$, where $S_{\text {Tot }} \equiv S_{\text {rad }}+S_{\text {col }}$. This factor is only applied to the inelastic and pair production processes, so that the model expression for the total photon energy deposition coefficient becomes 13

$$
\begin{aligned}
\mu_{\mathrm{en}}=\sigma_{\mathrm{PE}} & +\int_{0}^{T_{\max , \text { in }}}\left(1-\frac{S_{\mathrm{rad}}}{S_{\mathrm{Tot}}}\right) f_{\mathrm{in}} \frac{d \sigma_{\mathrm{in}}}{d T^{\prime}} d T^{\prime} \\
& +\int_{0}^{T_{\max , \mathrm{pp}}}\left(1-\frac{S_{\mathrm{rad}}}{S_{\mathrm{Tot}}}\right) f_{\mathrm{pp}} \frac{d \sigma_{\mathrm{pp}}}{d T^{\prime}} d T^{\prime},
\end{aligned}
$$

where the upper limits on the integrations apply to the maximum allowed emitted electron energies for the respective processes. The energy deposition coefficient is described in detail in the X-Ray Data section of the NIST website [5].

\section{Transport Formulation}

In the present work, the general transport process is focused principally on the description of the penetration of a primary electron field along with secondary bremsstrahlung photons generated by electron-atom interactions. For electrons, an essentially one-dimensional formulation is developed with some reference given to the effects of radially symmetric scattering. While the electron propagation is determined by the stopping powers, the photons are assumed to be transported along the direction of travel of the electrons. Photon intensity is governed by the radiative transfer equation [14, which utilizes the calculated attenuation coefficients.

\subsection{Electrons}

A quantity of fundamental importance in describing the transport of electrons in matter is the maximum distance of travel, which is determined from the energy loss stopping powers calculated from the modified Bethe-Bloch and bremsstrahlung formulas in Eqs. (1) and (13). This quantity is referred to as the CSDA range $R(E)$. For a given energy, $E$, the CSDA range is defined as

$$
R(E) \equiv \int_{0}^{E} \frac{d E^{\prime}}{S_{\mathrm{Tot}}}=\int_{0}^{E} \frac{d E^{\prime}}{S_{\mathrm{col}}+S_{\mathrm{rad}}}
$$

The inverse of the $R(E)$ function, $R^{-1}(E) \equiv E(R)$, may be used to construct the variation of $d E / d x$ as a function of distance traveled by an electron of initial energy $E_{0}$. Such a function, $S\left(E_{0}, x\right)$, is analogous to the usual Bragg curve, but neglects straggling and measures energy loss as a function of distance traveled along the particle's path rather than perpendicular depth in a material. Straggling is accounted for after the incorporation of the statistics 
associated with scattering. The residual energy of an electron, at position $s$, is given by

$$
\begin{aligned}
W(s) & =\int_{0}^{R} S\left(E_{0}, x\right) d x-\int_{0}^{s} S\left(E_{0}, x\right) d x \\
& =E_{0}-\int_{0}^{s} S\left(E_{0}, x\right) d x, \quad s \leq R .
\end{aligned}
$$

For a beam of mono-energetic electrons, it is assumed that the maximum distance traversed is the CSDA range and straggling is neglected. However, the average distance of penetration along the beam axis direction is generally less than the CSDA range due to multiple scattering effects. The average deflection for a unit path length can be defined by the inverse mean free path $\left(\lambda_{\mathrm{tr}}^{-1}\right)$ [9] which yields

$$
\langle\cos \theta\rangle=\frac{d\langle z\rangle}{d s}=\exp \left[-\int_{0}^{s} \frac{d s^{\prime}}{\lambda_{\operatorname{tr}}\left(s^{\prime}\right)}\right]
$$

and

$$
\langle z(s)\rangle=\int_{0}^{s} \exp \left[-\int_{0}^{s^{\prime}} \frac{d s^{\prime \prime}}{\lambda_{\operatorname{tr}}\left(s^{\prime \prime}\right)}\right] d s^{\prime} .
$$

In general, $\langle x\rangle$ is defined to be the arithmetic average of the variable $x$. For electrons, $\langle z(R)\rangle$ is interpreted as an "effective range" or the axial distance at which $50 \%$ of the electrons have stopped and takes place at the $z$-value for which the transmission is 0.5 .

A further critical assumption is made by specifying a Gaussian distribution about $\langle z(R)\rangle$, where $\langle z(R)\rangle$ is the distance of peak electron population. In addition, it is assumed that practically all electrons are stopped at $z=$ $R(E)$. This Gaussian represents the variation in electron path length due to multiple scattering effects. By invoking the formula for half-width of the Gaussian distribution, a value for deviation, $\aleph$, is found as 15 .

$$
\aleph=\frac{R-\langle z(R)\rangle}{2 \sqrt{2 \ln 2}} .
$$

The resultant Gaussian function may be interpreted as the probability that an electron penetrates an axial distance $z$ within increment $d z$,

$$
P(z)=\frac{1}{\aleph \sqrt{2 \pi}} \exp \left\{-\frac{[z-\langle z(R)\rangle]^{2}}{2 \aleph^{2}}\right\} .
$$

Integration of the Gaussian function results in an error function prescribing the number of particles having stopped over distance $z$. The complementary error function [16] results in the corresponding transmission function,

$$
\eta(z)=0.5\left\{1-\operatorname{erf}\left[\frac{z-\langle z(R)\rangle}{\aleph \sqrt{2}}\right]\right\} .
$$

For simplicity, a special algorithm for the standard error function has been utilized in the present code [17.

In addition to specifying the transmission probability of electrons as a function of axial penetration distance $z$, it is necessary to evaluate the variation of primary electron energy as a function of $z$. A formula has been developed [10] in terms of the moments of the mass scattering power that expresses, in cylindrical coordinates $(z, r)$, the distance covered for an average electron trajectory having axial distance $z$ and deflection radius $r$. The moments are calculated as

$$
a_{i}=\int_{0}^{z} P_{s}(u)(z-u)^{i} d u
$$

with $P_{s}$ given by Eq. (17). The moments are then used to derive an equation [10] for distance along an average trajectory passing through $(z, r)$,

$$
s(z, r)=\int_{0}^{z}\left[1+a_{0}\left(z^{\prime}\right)+\left(\frac{r^{2}}{a_{2}(z)}-1\right) \frac{a_{1}^{2}\left(z^{\prime}\right)}{a_{2}\left(z^{\prime}\right)}\right]^{\frac{1}{2}} d z^{\prime}
$$

The quantity $\sqrt{a_{2}(z)}$ has been identified [10] as the halfwidth at half-height of the distribution of deflection radius at axial distance $z$. This observation leads to the inference that a "most typical" trajectory intersects the point $\left(z, \sqrt{a_{2}}\right)$. The third term in Eq. (44) then vanishes. An "average mean path" now becomes,

$$
\langle s(z)\rangle=\int_{0}^{z} \sqrt{1+a_{0}\left(z^{\prime}\right)} d z^{\prime} .
$$

Reference to Eqs. (39) and 45 shows that the relationship between the mean axial distance and average mean path is such that $\langle z\rangle \leq z \leq\langle s\rangle$. An average residual energy $\langle W\rangle$ may be associated with an average distance along a path length $\langle s\rangle$ by again interpolating on a table of CSDA range-energy relations. However, the interpolated value $\langle W\rangle$ reaches zero before $z=R$ is attained because $\langle s\rangle$ is a value that includes average deflection and $z=R$ is the axial distance traveled in the continuous slowing down approximation assuming no angular deflection. The axial penetration, however, may extend beyond the distance at which $\langle W\rangle$ vanishes due to energy straggling. A provision for energy straggling is supplied by introducing a power law function of the form

$$
\langle W\rangle=A_{w}(R-z)^{k} .
$$


This formula is invoked somewhat arbitrarily for $z$-values for which $\langle W\rangle \leq 0.5 W_{\mathrm{CSDA}}$. When this condition pertains, the constants $A_{w}$ and $k$ are evaluated by point-slope matching at the $z$-value where $\langle W\rangle=0.5 W_{\mathrm{CSDA}}$.

In going from the boundary to distance $z$, particle number conservation requires that $\varphi\left(0, T_{0}\right) \delta T_{0}=\varphi(z, T) \delta T$, where the energy scaling corresponds to that prescribed by the CSDA range-energy relation for the material. In general, $\delta Y$ is defined as a small, finite difference in the value of $Y$. The above transport parameters, along with particle number conservation, may be used to devise an expression for the differential flux spectrum of electrons, $\varphi(z, T)$, at distance $z$ with kinetic energy $T$, given the initial spectrum at $z=0$ with initial kinetic energy $T_{0}$, $\varphi\left(0, T_{0}\right)$ :

$$
\varphi(z, T)=\frac{\varphi\left(0, T_{0}\right) \eta(z) S\left(T_{0}\right)}{S(T)} .
$$

In addition, the transmission function $\eta(z)$ is used to account for particles not arriving at $z$ due to interaction not accounted for in the stopping power. Division of the kinetic energy increments by distance increments leads to an expression in terms of stopping powers in Eq. (47). Thus, for a slab of thickness $w$, the differential spectrum of primary electrons may be found at any axial distance $z \leq w$.

If primary electrons are the only concern, the calculation could end with Eq. 47). However, bremsstrahlung photons are generated during the transport process. In order to take this process into account, a spatial grid is established within the slab layer. In the present code, a slab layer is assigned a spatial grid, normally of 20 to 30 points, with spacing increasing monotonically according to the following formula,

$$
d_{i}=\left(\frac{i-1}{N-1}\right)^{2} w
$$

Here, $d_{i}$ is the depth in the material slab of the $i$ th point and $N$ is the total number of points in the spatial grid of the slab. The photon source term may then be calculated at each spatial grid point as

$$
\zeta\left(E_{\nu}, d_{i}\right)=\int_{E_{\nu}}^{T\left(d_{i}\right)} \varphi_{e}\left(T^{\prime}\right) \frac{d \sigma_{\nu, e}}{d E_{\nu}} d T^{\prime} .
$$

Finally, the primary electron spectra provided by the present code are calculated at each spatial grid point as

$$
\varphi_{e, i}\left(d_{i}, T\right)=\frac{\varphi\left(0, E_{0}\right) \eta\left(d_{i}, E_{0}\right) S\left(E_{0}\right)}{S(T)} .
$$

Implementation of Eq. 47) for the illustrative low Earth orbit (LEO) spectrum [18 normally incident on aluminum provides the results shown in Fig. 1 (the environment spectrum is labeled $\mathrm{Z}=0.0$ ). The stopping of low energy electrons and the transfer of high energy electrons to lower energies is evident in the spectral functions at increasing penetration depths. Similar results for Jovian trapped

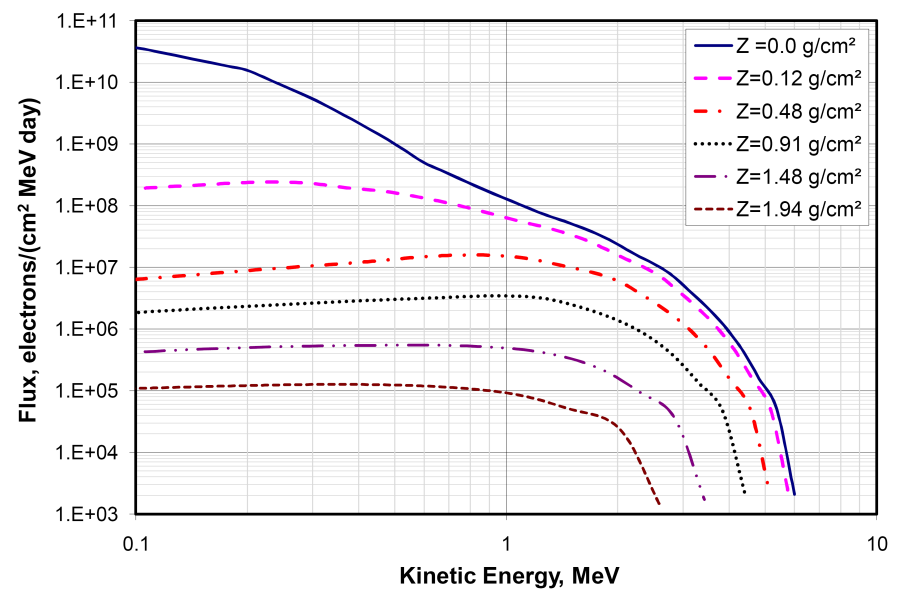

Figure 1: LEO electron spectra at several aluminum thicknesses.

electrons in the vicinity of Europa in aluminum are shown in Fig. 2 using an environment from the NASA Jet Propulsion Laboratory (JPL) Galileo Interim Radiation Electron (GIRE) Model [19]. The much greater penetration and more gradual attenuation is clearly evident for this very high energy environment. The peak in the flux occurs because low energy electrons are more likely to be stopped in aluminum which appears as a decrease in flux at low energies. High energy electrons, on the other hand, will be shifted to lower energies but not lost from the spectrum which leads the shift in the peak of the flux to higher energies with a decrease in peak flux.

\subsection{Photons}

In the previous section on cross section evaluation, it was shown that a spectral source term for bremsstrahlung photons may be calculated. See Eq. (12). When the differential electron flux spectra is specified as a function of

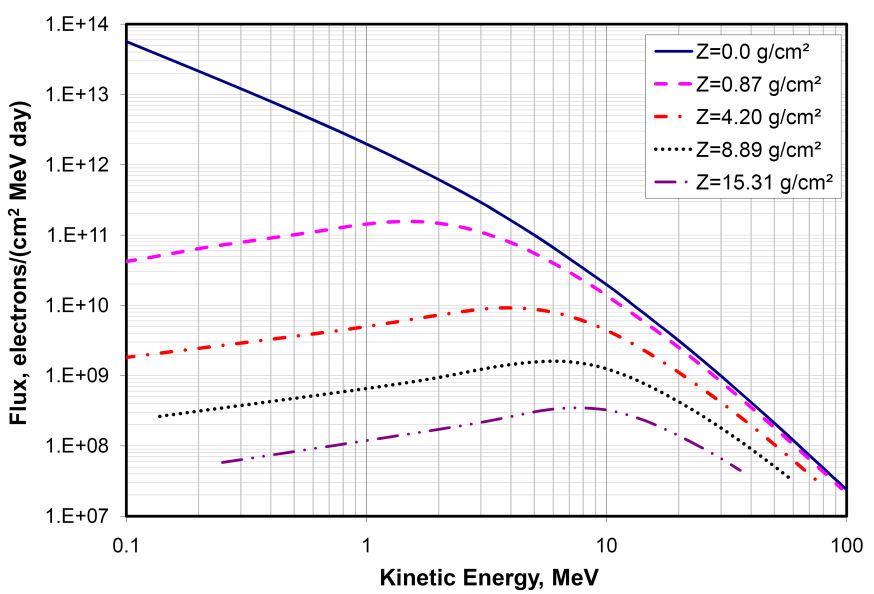

Figure 2: Europa electron spectra at several aluminum thicknesses. 
axial distance $z$, a corresponding photon flux may be determined using a solution of the radiative transfer equation for an emitting-absorbing medium [14. In addition to the source function, the total attenuation coefficient is required. The total attenuation coefficient is the sum of the relavant cross sections,

$$
\sigma_{\mathrm{Tot}}=\sigma_{\mathrm{PE}}+\sigma_{\mathrm{in}}+\sigma_{\mathrm{pp}} .
$$

The photon flux spectrum at each spatial grid point $d_{i}$ then involves integration of the source term multiplied by the attenuation factor;

$$
\varphi_{\nu, i}\left(d_{i}, E_{\nu}\right)=\int_{0}^{d_{i}} \zeta\left(x, E_{\nu}\right) \exp \left[-\sigma_{\operatorname{Tot}}\left(d_{i}-x\right)\right] d x .
$$

Photon spectra for the case of the Europa spectrum are illustrated in Fig. 3 and correspond to the electron spectra Fig. 2. The persistence of higher energy photons at large aluminum depths is indicative of their highly penetrating nature. The minimum and maximum values of the flux at low energies are due to the discontinuity in the photoelectric absorption cross section.

\subsection{Positrons}

Ordinarily, exposure effects due to space environment electrons may be adequately evaluated from specified electron and photon flux values at a given target location. The pair production contribution to the energy absorption coefficient should provide a fair estimate of exposure due to positrons. However, if details of specific positron interactions are of interest, it becomes necessary to include explicit transport of the positrons and their associated annihilation photons. In the present code, source terms and flux for positrons are evaluated, along with source terms for the annihilation photons, in an uncoupled manner with a view toward allowing the user to assess the relative importance of the presence of positrons. The positron source term, $\zeta\left(E_{+}, x\right)$, has already been given as Eq. (34). These antimatter particles have a "sink" term by annihilation with the constituent bound electrons of the medium. This term may be represented as 8

$$
N_{e} \sigma_{\mathrm{ann}}(\tau)=\frac{N_{e} \pi r_{e}^{2}}{\tau+2}\left[\frac{\tau^{2}+6 \tau+6}{\tau(\tau+2)} h_{1}-h_{2}\right],
$$

where $N_{e}$ is the number of electrons per unit mass of material and $\sigma_{\text {ann }}(\tau)$ is the total annihilation cross section, with

$$
\begin{aligned}
& h_{1}=\ln [\tau+1+\sqrt{\tau(\tau+2)}] \\
& h_{2}=\frac{\tau+4}{\sqrt{\tau(\tau+2)}}
\end{aligned}
$$

and $\tau$ representing the positron kinetic energy in rest mass units $(0.511 \mathrm{MeV})$.

Positrons initially at spatial location $x_{i}$ arriving at $x_{i}+$ $\delta x$ with energy $E_{j}$ began traversal of this increment at a higher energy, $E_{0}=E(R+\delta x)$, where $R$ is the residual range at $x_{i}+\delta x$. The CSDA process over the interval $\delta x$, with no loss of positrons requires that

$$
\varphi\left(E_{0}, x_{i}\right) S\left(E_{0}\right)=\varphi\left(E_{j}, x_{i+1}\right) S\left(E_{j}\right) .
$$

When annihilation takes place, the fraction of positrons from the incident spectrum lost in distance $\delta x$ is

$$
A_{T}(\delta x)=\exp \left\{-\int_{0}^{\delta x} N_{e} \sigma_{\text {ann }}\left[E\left(R_{j}+x^{\prime}\right)\right] d x^{\prime}\right\} .
$$

The positrons transmitted through interval $\delta x$ is then

$$
\varphi^{(t)}\left(E_{j}, x_{i}+\delta x\right)=\rho_{S} A_{T}(\delta x) \varphi\left[E\left(R_{j}+\delta x\right), x_{i}\right],
$$

with

$$
\rho_{S}=\frac{S\left[E\left(R_{j}+\delta x\right)\right]}{S\left(E_{j}\right)} .
$$

Additional contributions to the positron flux at $x_{i+1} \equiv$ $x_{i}+\delta x$ arise from the distributed sources in the interval that must include an additional annihilation factor, $A_{S}$;

$$
\varphi^{(s)}\left(E_{j}, x+\delta x\right)=\int_{0}^{\delta x} \zeta\left[E\left(R+x^{\prime}\right), x_{i}+x^{\prime}\right] A_{S}\left(x^{\prime}\right) d x^{\prime},
$$

with

$$
A_{S}\left(x^{\prime}\right)=\exp \left\{-\int_{0}^{x^{\prime}} N_{e} \sigma_{\mathrm{ann}}\left[E\left(R_{j}+x^{\prime \prime}\right)\right] d x^{\prime \prime}\right\} .
$$

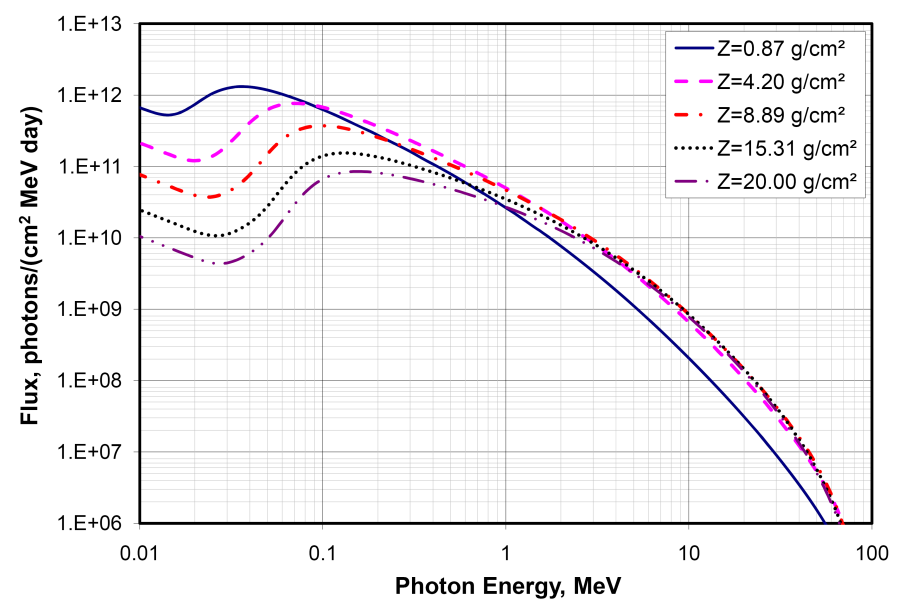

Figure 3: Photon spectra from Europa electrons [19] at several aluminum thicknesses. 


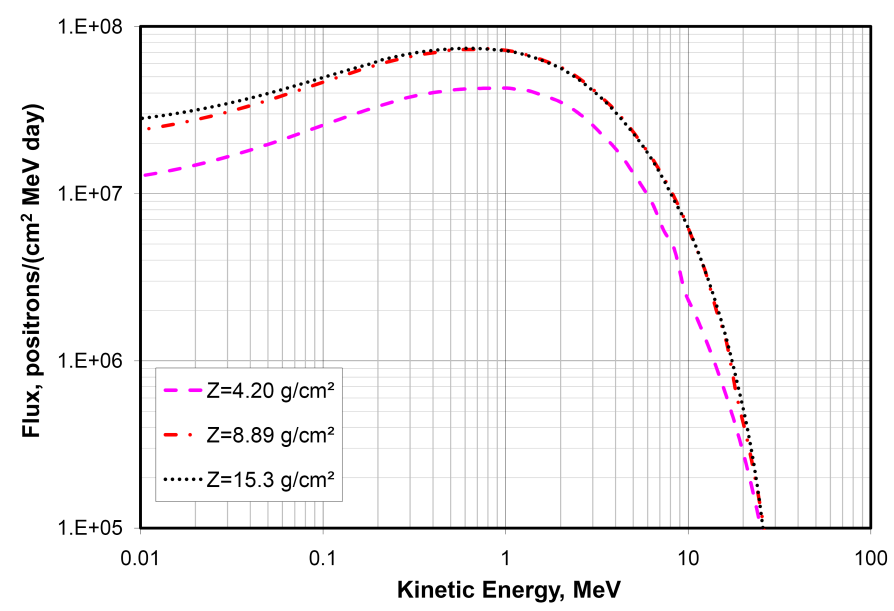

Figure 4: Positron flux at selected thicknesses in aluminum for Europa spectrum [19]. The curves for a depth of $8.89 \mathrm{~g} / \mathrm{cm}^{2}$ and $15.3 \mathrm{~g} / \mathrm{cm}^{2}$ overlap above $1 \mathrm{MeV}$.

Here, $A_{S}$ is the fraction of positrons lost from the positrons created in the distance increment.

The total positron flux, $\varphi_{e^{+}}=\varphi^{(t)}+\varphi^{(s)}$, is calculated in the code by numerical integration procedures applied to each of the established spatial grid intervals. Results for the Europa spectrum for selected thicknesses in aluminum are shown in Fig. 4 and may be compared with the corresponding photon flux of Fig. 3. The peak in the positron flux shown in Fig. 4 occurs at a positron kinetic energy of approximately $1 \mathrm{MeV}$. This peak in the positron flux is due to a minimum in the stopping power model at 1 $\mathrm{MeV}$ 6]. It is seen that the positron flux is a relatively small fraction of the governing photon field and that the uncoupled calculation is justified.

When the positrons are annihilated, photons are generated in accordance with the differential cross section [8].

$$
\frac{d \sigma_{\mathrm{ann}}}{d E_{\nu}}=\frac{\pi r_{e}^{2}}{\tau(\tau+2)}[q(\kappa)+q(\tau+2-\kappa)],
$$

where

$$
q(x) \equiv \frac{1}{x}\left(\tau+2+2 \frac{\tau+1}{\tau+2}-\frac{1}{x}\right)-1,
$$

with $\tau$ and $\kappa$ being the positron kinetic energy and the photon energy, respectively, in units of electron rest mass. The annihilation photons are restricted by kinematics to an energy range of

$$
\frac{m_{e}}{1+a^{\prime}} \leq E_{\nu, \text { ann }} \leq \frac{m_{e}}{1-a^{\prime}}
$$

where

$$
a^{\prime} \equiv \sqrt{\frac{\tau}{\tau+2}} .
$$

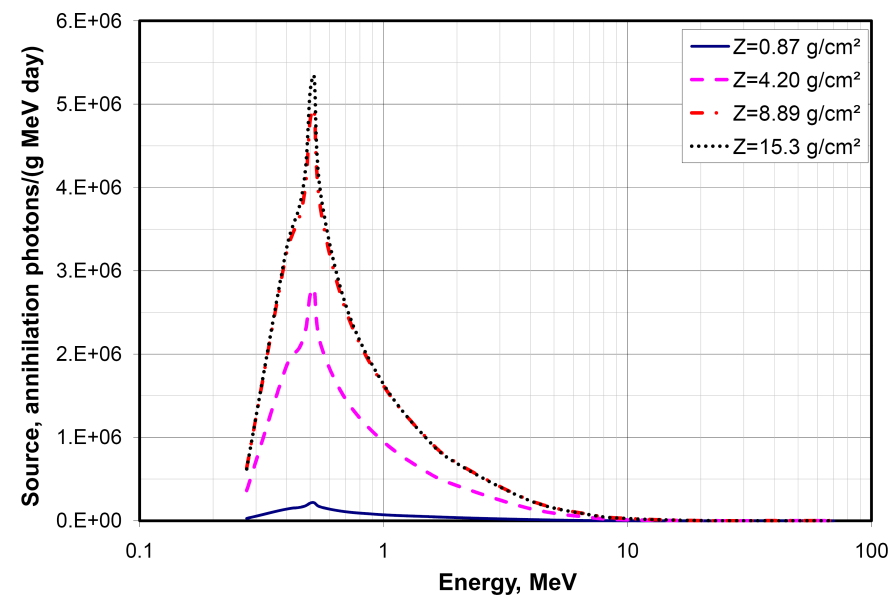

Figure 5: Annihilation photon source for Europa spectrum [19] in aluminum.

Finally, a source term for the annihilation photons per unit mass of material may be expressed as

$$
\zeta_{\nu, \text { ann }}\left(E_{\nu}, x\right)=\int_{T_{\min }}^{T_{\max }} \varphi\left(E_{+}, x\right) \frac{d \sigma_{\text {ann }}}{d E_{\nu}} d T_{+} .
$$

Production of annihilation photons occurs with the highest probability near the electron/positron rest mass energy. For the Europa case of Fig. 4, the annihilation photon source terms calculated according to Eq. (61) are shown in Fig. 5 and exhibit the expected peak at 0.511 $\mathrm{MeV}$. Since this contribution is small relative to the other processes, these photons are not transported in the present formulation.

\section{Dosimetric Calculations and Comparisons}

The conventional radiation dose for ionizing radiations in matter is defined as the energy imparted to a mass element of the material by the particles traversing the elemental mass. In general, only energetic charged particles are responsible for the energy transfer. The "dose" attributed to uncharged particles (e.g. photons, neutrons) results from the charged particles generated within the medium by the neutrals.

\subsection{Electron and Photon Dose Evaluation}

Energy deposition of electrons is calculated by multiplying the local flux (differential in energy) by the total stopping power and integrating over energy. The dose at a given location is given by

$$
D_{e}(x)=\int_{0}^{\infty} \varphi_{e}(x, E) S(E) d E .
$$


In Eq. (62), $E$ represents the electron energy at position $x$. The local differential flux, $\varphi_{e}$, has units of electrons $/\left(\mathrm{cm}^{2}\right.$ $\mathrm{MeV})$. The stopping power, $S$, has units of $\mathrm{MeV} \mathrm{cm}^{2} / \mathrm{g}$, while the units of $D_{e}$ are $\mathrm{MeV} / \mathrm{g}$ which may be converted to cGy or rads upon multiplication by the conversion factor $1.602 \times 10^{-8}$. For photons, absorbed dose is characterized by the energy deposition coefficient, $\mu_{\mathrm{en}}$, which is described in Eq. (35) and is expressed herein as a mass absorption coefficient with units $\mathrm{cm}^{2} / \mathrm{g}$. The dose attributed to photons is then

$$
D_{\nu}(x)=\int_{0}^{\infty} \mu_{\mathrm{en}} E_{\nu} \varphi_{\nu}\left(E_{\nu}, x\right) d E_{\nu} .
$$

The use of $\mu_{\mathrm{en}}$ to calculate effective photon dose (as is done in the present code) is a simplistic approximation method that has been widely used and considered to be an adequate representation of this dose contribution [2, 5]. This approximation assumes that all the energy deposited by photons is deposited by charged particles at the point of their production. A more direct evaluation would be to use the appropriate cross sections for generation of secondary electrons $\left(\sigma_{\mathrm{PE}}, \sigma_{\mathrm{in}}\right.$, and $\left.\sigma_{\mathrm{pp}}\right)$ to compute respective source terms in the manner expressed in Eq. (34). The corresponding flux terms could then be determined using procedures analogous to that described for the positron transport in Eqs. (53)-(57). Such modifications may be considered for future upgrades if the additional complexity appears to be warranted.

\subsection{Sample Calculations and Comparisons}

Calculations performed with the present code were selected to examine the behavior of trapped electron spectra interacting with light and heavy materials. The chosen

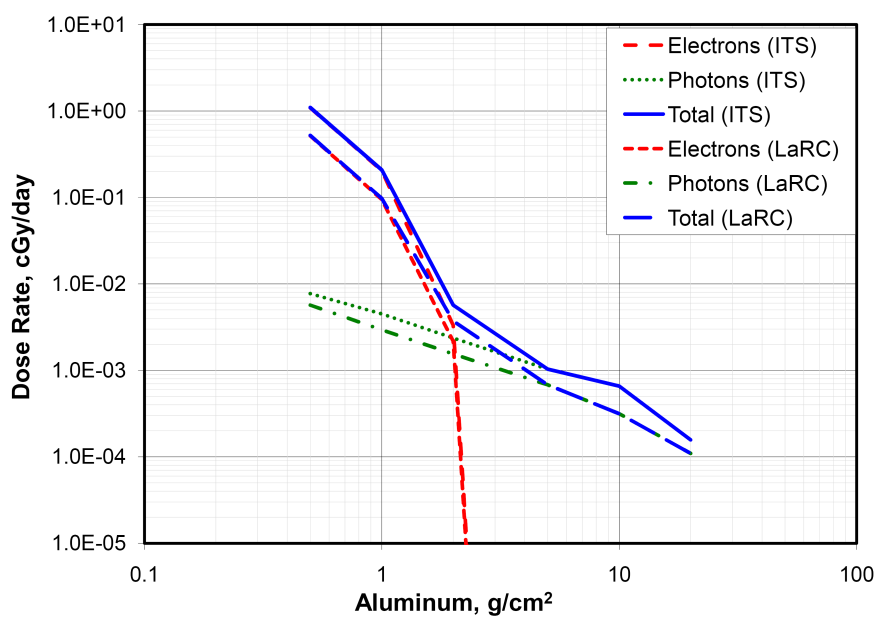

Figure 6: Comparison of LaRC results in aluminum with ITS 20] for LEO spectrum 18. The results for ITS and LaRC overlap for electrons above approximately $2 \mathrm{~g} / \mathrm{cm}^{2}$ of aluminum. electron environment spectra (boundary conditions) are shown in Figs. 1 and 2 and represent, respectively, the environment in LEO (400 km, 51 inclination) 18 and the environment in the vicinity of the orbit of the Jovian moon Europa 19. The initial environment is shown as the curves labeled $\mathrm{Z}=0.0$ in Figs. 1 and 2, along with the flux spectra at various depths in aluminum. After transport through the shield medium, the doses have been evaluated in silicon to simulate exposure of a solid state device.

The Integrated TIGER Series (ITS) Monte Carlo code 20] was used to calculate dose for the LEO spectrum electrons on aluminum at normal incidence for several depths. The identical scenario was used with the LaRC deterministic code and the results are compared in Fig. 6. The LaRC code gives generally lower values for the dose versus depth functions than the Monte Carlo results, but the functional behavior is very similar. Greater differences are to be expected for the low energy spectra of LEO because scattering processes are more prominent in the transport process and are treated differently in the two calculations.

For higher energy electron spectra, sample application comparisons have been made for a Jovian electron environment generated by the NASA-JPL GIRE model [19. The relevant spectrum is shown in Fig. 2 as the curve labeled $\mathrm{Z}=0.0$ and represents electron flux in Jupiter's equatorial plane at the average orbital distance of Europa. The normal incidence on a semi-infinite slab scenario was used for the materials aluminum, tantalum, and copper-tungsten (50\%-50\%) alloy. The previous LEO spectrum exhibited very few electrons above $5 \mathrm{MeV}$, whereas the Europa electrons may have substantial population up to and beyond $100 \mathrm{MeV}$.

The dose versus depth curves for the Europa spectrum are given in Figs. 7, 8, and 9 for the specified materials. In general, the LaRC deterministic calculations show improved agreement with the corresponding Monte Carlo

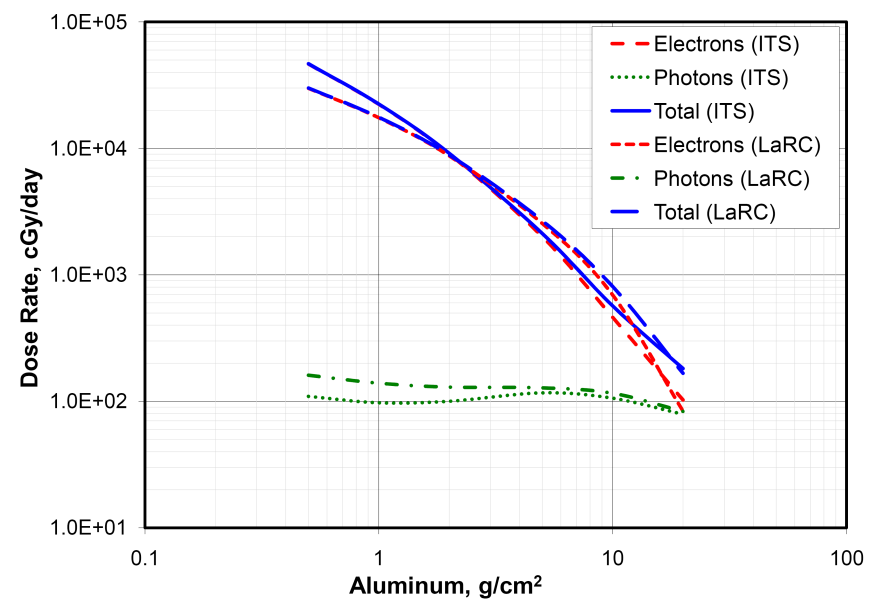

Figure 7: Comparison of LaRC results in aluminum with ITS 20] for Europa spectrum 19. 


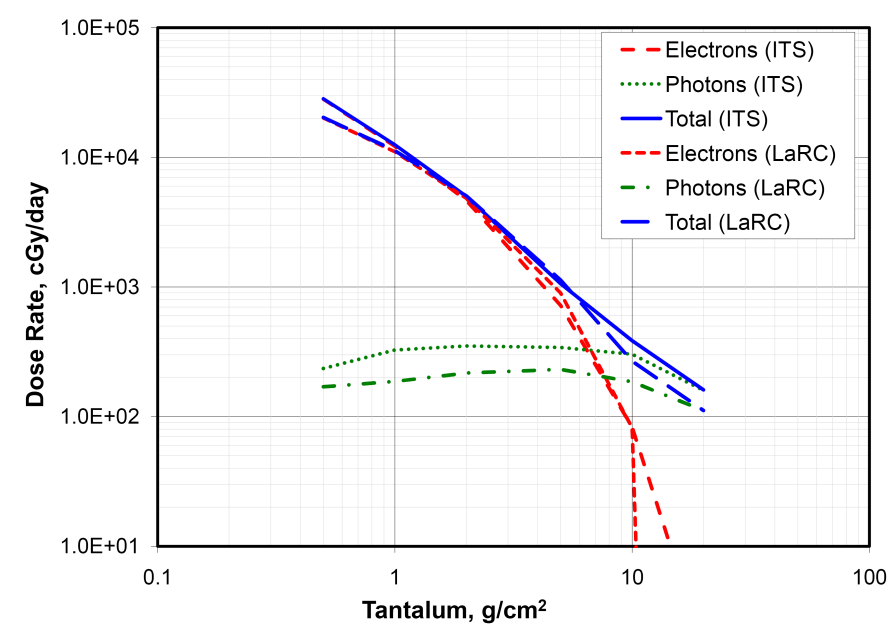

Figure 8: Comparison of LaRC results in tantalum with ITS [20] for Europa spectrum [19].

results compared to the lower energy LEO environment. The range of scaled thickness for the Europa cases is from 0.5 to $20 \mathrm{~g} / \mathrm{cm}^{2}$. It is seen that electron stopping is practically complete at the end of this thickness range, after which the bremsstrahlung contribution dominates.

\section{Concluding Remarks}

The electron/photon transport code described here has been developed with a view toward simplicity and speed in analysis of exposure from space environment electrons. In its present form, the code may be implemented to great advantage in shield material trade studies, numerical and statistical experiments, uncertainty analyses, etc., despite some observed differences with Monte Carlo comparisons. Numerous comparative calculations, in addition to those reported here, have been performed with similar degrees of agreement as those presented in the text. In particular, calculations for the trapped Jovian environment compare more favorably with corresponding Monte Carlo results than do the comparisons for the much lower energy-range spectra of LEO. This fact suggests that the deterministic formulas for low-energy scattering should be improved in future versions.

There are several areas in which improvements to the code are being considered. The various cross section comparisons with NIST [5] calculations show several instances where the LaRC code cross sections may be improved by introducing selective correction terms, even though the cross section deviations noted have little impact on final exposure results. With regard to transport, the present code is amenable to immediate extension to a twodimensional axi-symmetric representation. In addition, more explicit details pertaining to very high energy processes involving positrons and their annihilation photons may be obtained by a formal coupling of these species to

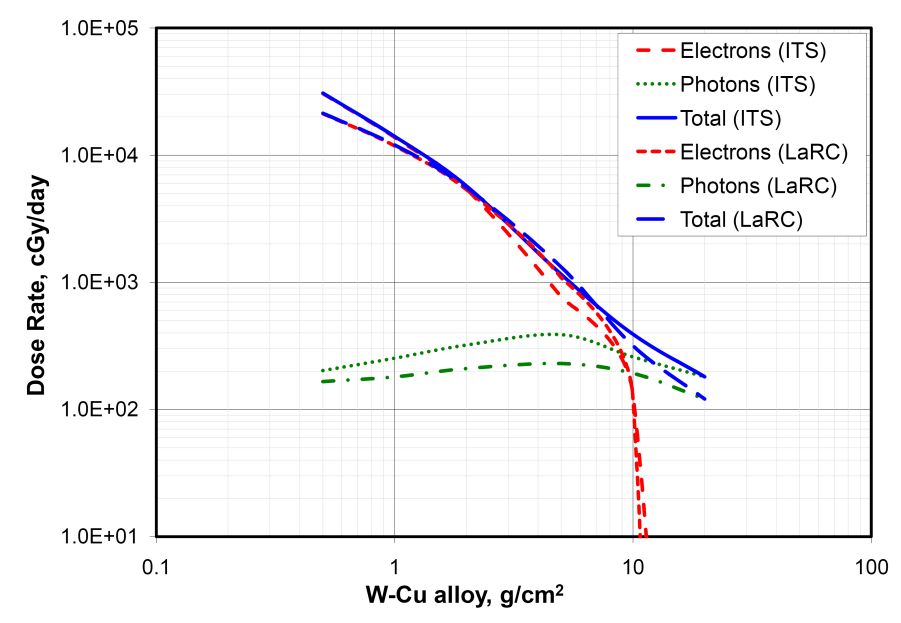

Figure 9: Comparison of LaRC results in W-Cu alloy with ITS 20] for Europa spectrum 19.

the general electron/photon transport. Although no significant errors are indicated as a result of the decoupling of positron and annihilation photon transport for the Jovian spectral environments, use of the present code for high energy beam simulation or cosmic ray shower analysis would most likely require full coupling of these processes. It is therefore natural to anticipate code upgrades and extensions in the near future.

\section{References}

[1] J. W. Wilson, F. F. Badavi, F. A. Cucinotta, J. L. Shinn, G. D. Badhwar, R. Silberberg, C. H. Tsao, L. W. Townsend, R. K. Tripathi, NASA TP-3495, National Aeronautics and Space Administration (1995).

[2] D. W. Anderson, Absorption of Ionizing Radiation, University Park Press, Baltimore, 1984.

[3] R. M. Sternheimer, R. F. Peierls, Phys. Rev. B 3 (11) (1971) 3681-3692.

[4] P. Sigmund, Phys. Rev. A 54 (4) (1996) 3113-3117.

[5] National Institute of Standards and Technology (NIST), http: //www.nist.gov accessed August 4, 2009.

[6] J. N. Nealy, C. K. Chang, R. B. Norman, S. R. Blattnig, F. F. Badavi, A. M. Adamczyk, NASA/TP-2010-216168, National Aeronautics and Space Administration (2010).

[7] H. W. Koch, J. W. Motz, Rev. Mod. Phys. 31 (4) (1959) 920955.

[8] I. Kawrakow, D. W. O. Rogers, PIRS-701, National Research Council Canada Report (2009).

[9] D. Liljequist, M. Ismail, Journal of Applied Physics 62 (2) (1987) 342-350.

[10] I. A. D. Bruinvis, W. A. F. Mathol, P. Andreo, Phys. Med. Biol. 34 (4) (1989) 491-507.

[11] X. A. Li, D. W. O. Rogers, Electron mass scattering powers: Monte Carlo and analytical calculations, Med. Phys. 22.

[12] J. W. Motz, H. A. Olsen, H. W. Koch, Rev. Mod. Phys. 41 (4) (1969) 581-639.

[13] C. Leroy, P.-G. Rancoita, Principles of Radiation Interaction in Matter and Detection, World Scientific, New Jersey, 2004.

[14] S. Chandrasekhar, Radiative Transfer, Dover Publications, New York, 1960.

[15] S. Brandt, G. Cowan, Data Analysis, Springer-Verlag, New York, 1999. 
[16] G. B. Arfken, H. J. Weber, Mathematical Methods for Physicists, Academic Press, San Diego, 2001.

[17] S. Winitzki, A handy approximation for the error function and its inverse, http://homepages.physik.uni-muenchen.de/ Winitzki/erf-approx.pdf, accessed August 4, 2009.

[18] SPENVIS: The European Space Agency (ESA) SPace ENVIronment System, http://www.spenvis.oma.be/ accessed August 4, 2009.

[19] H. B. Garrett, I. Jun, J. M. Ratliff, R. W. Evans, G. A. Clough, R. W. McEntire, JPL Pub. 03-006, Jet Propulsion Laboratory (2003).

[20] J. Halbleib, R. Kensek, G. Valdez, T. Mehlhorn, S. Seltzer, M. Berger, SAND91-1634, Sandia National Laboratory (1992). 